\title{
NS2 Based Simulation Tool for Performance Evaluation of TCP Traffic in Different Networks
}

\author{
Kumaraswamy Gajula, Prasad Janga
}

\begin{abstract}
Execution appraisal of TCP website traffic in OBS frameworks has been underneath heightened examine, due to the fact TCP builds up the majority of net visitors. As a dependable and transparently open take a look at framework, ns 2 has been generally used for considering TCP/IP frameworks; anyways ns 2 dreams an extensive parcel of the elements for imitating optical burst buying and selling frameworks. in this paper, a ns2 primarily based OBS reenactment gadget (nOBS), that is labored for reading burst assembling, reserving and strife targets counts in OBS frameworks is proven. The middle factor and association inquiries in OBS are connected in $\mathrm{nOBS}$ for making optical facilities and optical institutions. The passage, attention and flight middle factor functionalities are joined into a run of the mill optical center factor constructing, which incorporates masters chargeable for burstification, controlling and arranging. The results of burstification parameters, e.G., burstification break, burst size and number of burstification backings enhance center thing, on TCP execution are investigated the usage of nOBS for one among a type TCP interpretations and unmistakable framework topologies. The path of motion of libraries we long-established for this object is called Multi InteRfAce move Layer Extension for ns2 (MIRACLE). They replace the functionalities supplied with the aid of the network Simulator ns 2 by using way of giving a beneficial and embedded engine for coping with skip-layer messages and, on the equal time, enabling the combination of various modules inside every layer of the display stack. as an instance, various framework, association, MAC or physical layers may be resolved and used inside a comparative center. the consequences of this are puzzling. As an trouble of first importance, the structure empowers the execution and the reenactment of present day-day correspondence structures in ns 2 . moreover, due to its planned great, the code is probably reduced, re-usable and extensible.
\end{abstract}

Record Terms-MIRACLE-Multi InteRfAce cross Layer Extension, GLL-commonplace hyperlink Layer, MRRM-Multi Radio aid control.

\section{INTRODUCTION}

Over the maximum present day couple of years, progresses within the tool for far flung structures management and, specially, inserted chip advances have made it viable to provide extraordinarily little radio belongings and not using a trouble. This empowers the aggregate of severa advances in a similar portable hardware. those multi-innovation preparations are currently handy to be had and open up the chance of abusing new correspondence requirements. As multi-interface gadget ultimately finally ends up on hand effortlessly, there may be a parallel requirement for data its exhibition points of confinement and contriving new systems manipulate conventions as a manner to utilize the furnished functionality. frequently, those frameworks are too problematic to even take into account being absolutely described systematically, and we need to turn to precise reenactment devices for his or her familiar comprehension. one of the maximum finished workout gadgets in the structures administration inquire about network is without uncertainty the community Simulator, ns2 [2]. We watch, anyhow, that ns2 does now not as of now bolster severa radio interfaces and needs adaptable gadgets for the movelayer control of correspondence frameworks. besides, inside the well-known go together with the drift of the check machine, the far flung channel is spoken to with the beneficial aid of ridiculous fashions, which may also set off one-sided effects. additionally, non-compulsory utilization of the far flung channel are handy for explicit radio advances, for example, Bluetooth. All things taken into consideration, the ones are neither institutionalized nor reusable for various radio interfaces. This makes it hard to finish investigations on faraway conjunction and variety sharing. in this paper we gift a layout that we produced for the ns2 test system as a way to fill those holes. Our form is called Multi InteRfAce go Layer Extension (MIRACLE) for ns2 [3]. it's miles imagined as a spread of dynamic libraries that are stacked to embody guide for multi-innovation and pass-layering. We likewise composed a restore which evokes using dynamic libraries in ns2 (Fig1). Strikingly, going for walks with dynamic libraries permits the improvement and resulting usage of latest highlights with out the requirement for re-aggregating the entire check device. fact be informed, libraries may be stacked on interest at undertaking time. furthermore, as we display later, our engineering is highly specific because it lets in the interconnection of numerous down and upstream modules at each layer within the conference stack.

Revised Manuscript Received on September 10, 2019.

Kumaraswamy Gajula, Assistant Professor, Department of Electronics and Communication Engineering, CMR Institute of Technology, Hyderabad, Telangana, India.

(Email: kumaraswamygajula @gmail.com)

Dr. Prasad Janga, Professor, Department of Electronics and Communication Engineering, CMR Institute of Technology, Hyderabad, Telangana, India.

(Email: prasadjanga85@gmail.com) 


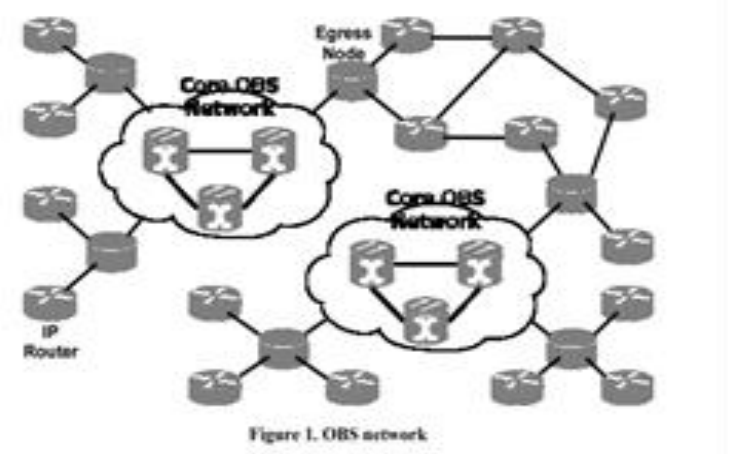

When further designing is done then his communication method between Network Simulator and Traffic simulator is maintained (Fig.2.)

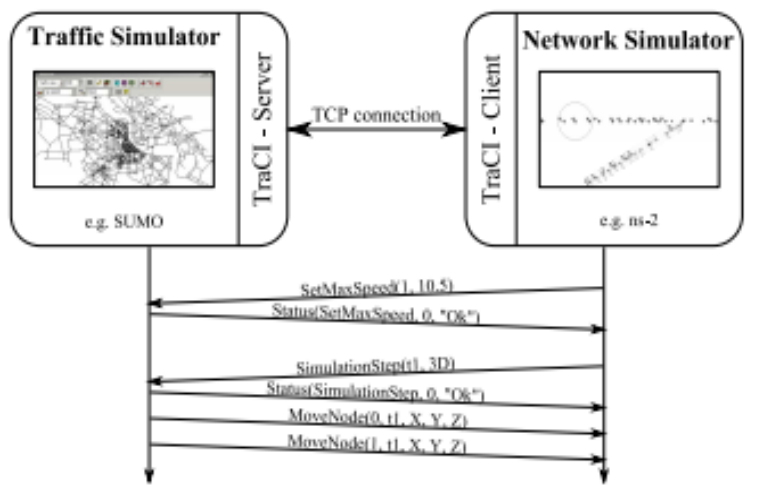

Figure 2. Framework engineering and an activity case of the order reaction trade between the system and the traffic test system displayed as a course of events outline.

\section{ALGORITHM USED}

A Modular architecture for Multi technology help in wireless structures in Fig. 3, we document a graph demonstrating the convention engineering for an Ambient Networks empowered terminal. For illustration, UMTS and IEEE 802.eleven radio upgrades are plotted in the decide. A elegant IP-empowered convention stack is carried out as a starting diploma. what's greater, we represent diverse modules (appeared within the right-half of of of of the figure) which include the Ambient Networks perception. as an instance, those modules are in price of starting a CA among the flexible substance and the chose get right of entry to component (AP), to show the network recognition of the terminal, to change the far flung innovation getting used, and so forth. Encompassing Networks modules are alluded to as beneficial Entities (FEs). The widespread link Layer FE (GLL in the A wording) is an adjustment layer this is mediated between the IP layer and the innovation ward layers, i.E., the hyperlink Layer (LL) and the physical layer (PHY). Its principle method is to enhance modern functionalities at the connection layers of the claimed radio improvements. as an instance, because of the GLL one ought to exchange/display screen LL parameters, consist of new highlights, for example, Hybrid ARQ calculations, parcel based totally beforehand mistake redress (for stepped forward multicast execution), and so forth. what is more, as regarded in Fig. three the GLL is accountable for getting QoS pointers for every the MAC and the PHY layers. those markers are both gotten thru the gathering and the following elaboration of the ads despatched through the APs or from the accumulation of measurements, for instance, bit blunder charge and have been given control, sooner or later of information transmission/accumulating. exquisite markers might be explicitly diagnosed with the gotten energy or be client characterised (showed, in Fig. 3, thru Pow/SINR and QoS, one after the other). the ones high-quality markers are then passed to the Multi Radio resource control FE (MRRM). MRRM carries the network industrial FE (NAD) and an execution purpose it clearly is MRRM specific. The MRRM can be seemed due to the truth the center of the A format: it settles on options on the APs to enroll in and the radio advances to apply every time, it devices parameters to carry out power reserve price range and, all in all, to streamline the presentation for the right now applied tool interfaces. In element, the project of the NAD is to resolve drawing near advertisements (from neighboring APs) clearly as to place the notices to be despatched with the resource of using the terminal inside the privilege an commercial enterprise employer. All FEs are related to the usual delivery Layer Protocol (GTLP), this is the car convention implemented for between terminal A correspondence. notice that the MRRM (or better its execution reason) is additionally legitimately related to the convention stack. mainly, the execution intent of the MRRM is legitimately appended to the connection layer (via GLL). this is to cope with tool association techniques, (as an example, wi-fi association messages) whilst IP availability regardless of the reality that want to be built up. Likewise, LL messages are required through Ambient community Attachment processes, which might be crucial to accumulate essential availability with a much flung tool. these messages are moreover overseen by way of the usage of the MRRM execution cause. To aggregate up, there are degrees of correspondence. The primary (and least complicated) stage endeavors LL availability, and is applied to send notices (from APs), set up connection needs (from flexible terminals), to attach a portable hubs with a few distinct system and, at lengthy final, to execute Ambient Networks connection strategies. The $2 \mathrm{~d}$ sort of correspondence, that could be a unique, takes place through the GTLP, this is responsible for trading A messages among various physical factors (e.G., terminal and AP). in any case, this ultimate form of correspondence requires IP availability and therefore can't be overseen at the connection layer. The final element inside the design is the Comp-FE, which handles Composition structures. CompFE is related to the execution purpose within the MRRM and conveys to mix Comp-FEs via the GTLP. form isn't always dealt with in detail on this paper, for additonal data the in step with man or woman is alluded to [24, 25]. Tables collectively with at gift joined APs (or systems), evaluating QoS and the kind of Composition this is right now dynamic for each considered one of them are located away on the MRRM and on the Comp-FE. We see that this structure is really extensive, and can be efficaciously reached out by way of which incorporates extra FEs. At prolonged very last, examine that

Published By: 
the A expertise responds to triggers from the software program program layer, which units QoS reference profiles and shows whether or no longer the client is really fulfilled approximately the plain high-quality. We strain that in this paper we center spherical a particular A format this is, be that as it may, a protracted way from being completed or agent of the whole Ambient Networks structure. inside the A undertaking, reality be advised, hundreds more FEs are being characterized and similarly angles, for instance, validation, charging, protection, media server assist, and so forth., are taken into consideration additionally. the ones are usually performed as FEs and characteristic all round characterized jobs and interrelations. on this paper, we as an alternative cognizance at the insignificant diploma of detail we need to determine handover strategies in structures such as awesome improvements.

PRRP varies from modern-day conventions, as an instance, LEACH and CELRP from a couple of factors of view. This examination anticipate that hubs recognise approximately their land regions in WSNs the use of GPS or some different savvy vicinity opinions [50, 75-79]. every hub can employ severa frequencies for transmitting and getting as it's miles large that sensor hub has a multichannel handset. it is time-honored that each hub can transmit its statistics to the sink as hub has capability to transmit for longer separation.

All hubs have homogeneous strength degree at starting stage and this supposition this is usually done in writing [80]. Sensor hubs are circulated inside the lattice institution with a uniform arbitrary style and the sink is set in the focal element of sensor put together. This supposition this is mainly relevant for timberland fire statement frameworks and calamity the board frameworks.

\section{NETWORK MODEL}

The low-cost tool version for PRRP execution has the layout as seemed in parent 1.it's far considered as a haphazardly uniform conveyed WSN in which hubs are circulated arbitrarily in a sensor area relying on a community role. The static sink is placed inside the focal issue of the machine. The sensor hubs inside the gadget are isolated into diverse gatherings of bunches on every side of the sink. usually, the ranges are characterised in radii at some point of the sink extending interior degree Dzero, degree $D 1$, and plenty of others, contingent upon the tool length. every degree covers a couple of matrix (incompletely or absolutely) and has a realistic range wherein the amount of hubs lies, that is relying upon the dispersion of the hubs. before the whole lot, a sign with energy degree Ezero is transmitted from the sink into the gadget. The sign with electricity $E 0$ might be gotten especially via using those hubs positioned close to the sink. subsequent to tuning in to the signal, the ones hubs will react to the sink and they may be enrolled as tier $D 0$ hubs. next, the sink will sell sign with transmission power of $E 1$, in which $E 1>E z e r o$. The hubs, aside from $D 0$, will react to this signal, shaping level $D 1$ hubs. This technique may be rehashed until a preset quantity of tiers are framed

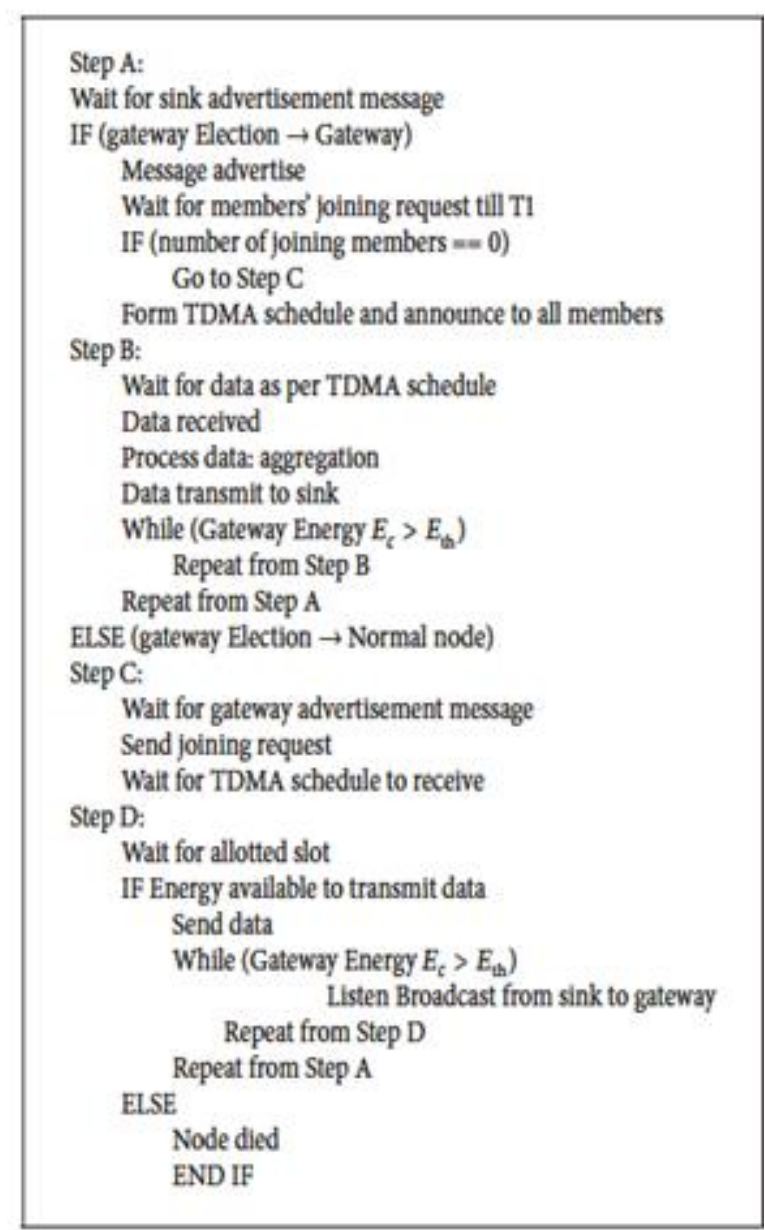

Figure.3. Algorithm used

\section{ENERGY MODEL.}

The strength control model used in this evaluation paintings is predicated upon the possibility that essentialness use is direct relating to the transmission department. condition (1) under well-knownshows the middle point essentialness use for transmitting $k$ bits of facts for a department of $d$ meters. The imperativeness consumed for tolerating of $k$ bits is given with the aid of (2) [81]:

$E \mathrm{Tx}=k E$ elec $+k E \operatorname{ampd} 2$,

$E \mathrm{Rx}=k E$ elec

where Eelec is the electronics energy in transceiver and $E$ amp is the amplifier energy.

D3 D2 D1 D1 D2D0 sink/base station Cluster head

The base vitality for a hub to have the option to partake in the coming round of steering (or it is the base vitality to take an interest in next round) is surrendered (3) as Ethresholdmin:

$E$ thresholdmin $=k E$ elec $+k E$ ampd28kEelec.

\section{SIMULATION RESULTS}

Reenactment scenario to numerically survey the rightness and, at the same time, the practicality of our system, we installation a diversion situation as seeks after. We taken into consideration two radio tendencies, i.E., IEEE 802.11g 
and UMTS. Compact terminals are subjectively dispersed, at the begin of the diversion, internal a internet site of four hundred $\times$ four hundred $\mathrm{m} 2$. We take into account a lone UMTS AP, put inside the component of convergence of the area to provide incorporation to all middle elements. We besides viewed as a unmarried IEEE 802.11g AP, in like manner located in the component of convergence of the multiplication region and presenting incorporation to the terminals positioned inner a division of around one hundred m. All center factors are ready with both radio progressions and are bendy. For the physical flexibility, we grasped the Gauss-Markov compactness model [15] considering the two everyday paces of and $15 \mathrm{Km} / \mathrm{h}$. A schematic depiction of the multiplication state of affairs is confirmed up in Fig. 4. Terminals get UDP information site visitors from their mobile community Operator (MNO within the decide), that is placed inside the fixed internet little bit of the framework. The MNO is related with both UMTS and 802.11 APs through two committed burdened out channels. those are sans botch, fixed deferral (two hundred ms) channels. facts streams in the downlink heading (APs $\rightarrow$ terminals), but popular (e.G., AP connection, ARP, and plenty of others.) and A hailing messages use each uplink and downlink channels. Downlink information site visitors is bursty: in the course of every burst, statistics is despatched determinedly at a pace of $70 \mathrm{Kbps}$. In our diversions, we considered burst terms of five, 10,15, 20, 25 and $30 \mathrm{~s}$. The among burst destroy is relentless and it became set to 10 seconds for the diversion effects we file on this paper. For the radio get right of entry to the board direction of movement, we pondered the going with instances. As an evidence behind our display appraisal, we realized an Ambient Networks unmindful form, in which UMTS and 802.eleven exist together but quite fundamental guidelines are used to select out the radio access. In specific, for this example the radio improvement is picked at the beginning of each burst, in perspective on were given sign levels, and is stored unaltered till its forestall. As a resulting recreation plan, we taken into consideration an Ambient Networks cautious desire method, as proven via which available get entry to openings are constantly evaluated and handover among systems is viable at some thing issue. For this circumstance, the important authority engine is ready inside the MRRM and old fashion strategies are used to lessen stupid handovers and pingponging.four inside the clean access guarantee path of movement no pass is made while the information channel is dormant (i.E., the radio being utilized is surveyed and possibly changed apparently sooner or later of the social occasion of a burst of information), at the identical time as, within the AN-engaged technique, MRRM reliably video display units the evident institutions to be readied while the information will come. ultimately, in our proliferations giving off changed into not authorized, i.E., terminals ought to genuinely speak about clearly with any of the APs and couldn't manhandle various terminals as repeaters

Reenactment consequences are confirmed up in Fig. 5. In Fig. 5(a) we document the usual package bungle price that a terminal opinions at some degree inside the social occasion of a burst of statistics. both the easy and the AN-enabled get to assurance attracts near (see beyond location) are showed up inside the determine. precisely as anticipated, computations provide higher execution to all impact lengths. this is required to the cautious and endless evaluation of available receives to. in addition, we be aware that presentation will increase at decrease speeds $(2 \mathrm{Km} / \mathrm{h}$ within the determine). this is a end result of the way wherein that an extended pace identifies with a higher extensive style of handovers which, in this manner, might also incite a better bundle adversity. We note that, anyways, the development in the package goof fee is limited. Fig. 5(b) suggests the entire scale variety of control bytes transmitted by manner of an Ambient Networks terminal in the course of the social occasion of a burst of records. As an hassle of first importance, we see that a growing rate activates an extended visitors. the reason is a similar we noted above, i.E., a better handover repeat. besides, the manipulate site visitors overhead creates with the burst term. In all honesty, clients during longer affects will truly flow into out of attention of the serving AP. For this situation, they need to start another handover method, thusly handing over further manage traffic.

Comparative evaluation: consistent with Packet average consumed strength. on this assessment, PRRP and CELRP display are taken a gander at depending at the ordinary ate up essentialness in keeping with group as confirmed up in discern 6. it's miles determined from our recognitions that CELRP from the begin demonstrates fantastic execution for traditional imperativeness in line with package deal deal seemed in a different way in relation to PRRP. anyways, after a constrained functionality to middle time PRRP indicates a crucial development in it with the extended extensive variety of facts durations. Of path, whilst differentiating PRRP and CELRP and extra alters of data drift, it's far located that PRRP is extra gainful than CELRP. In PRRP, the cause at the back of constantly ordinary imperativeness ate up at the simple round is that the three early on levels are for direction of motion and there can be no facts transmission all through this. Thusly, the imperativeness utilized in these hidden ranges is seen as overhead essentialness, that is more than the essentialness stored in information transmission arrange for first period PRRP speaks to crucial for regular in step with package deal essentialness use in the wake of completing its whole round and in particular inside the wake of continuing with its couple of transmission periods with its critical form tree. parent 6 demonstrates the correlation of absolute wolfed electricity by means of diverse hubs in a powerful size of time. 


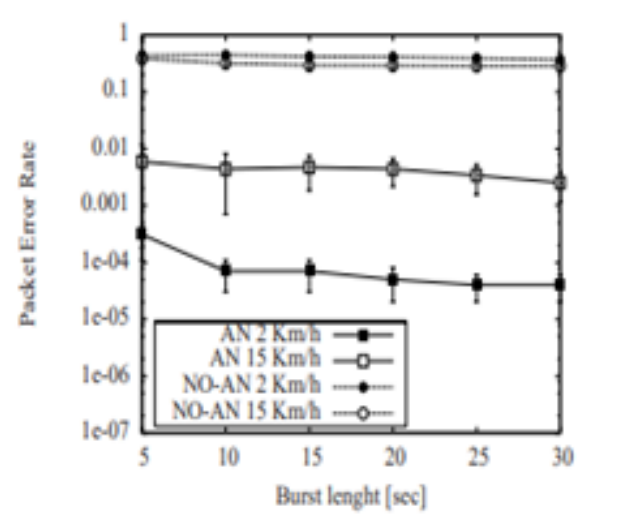

Figure.5 (a). Average packet error rate

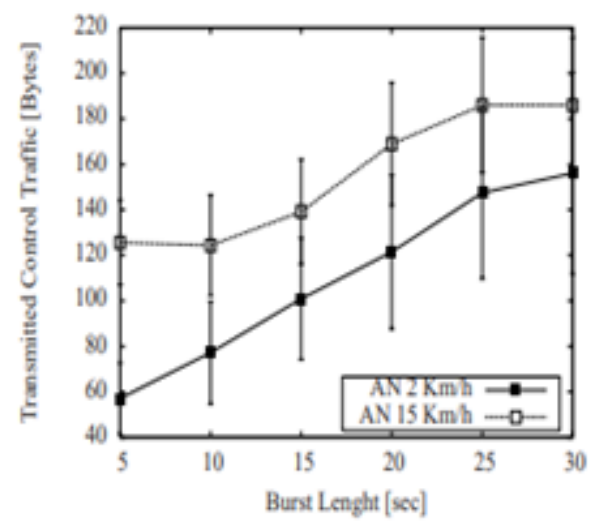

Figure.5 (b).Total Number of control bytes transfer

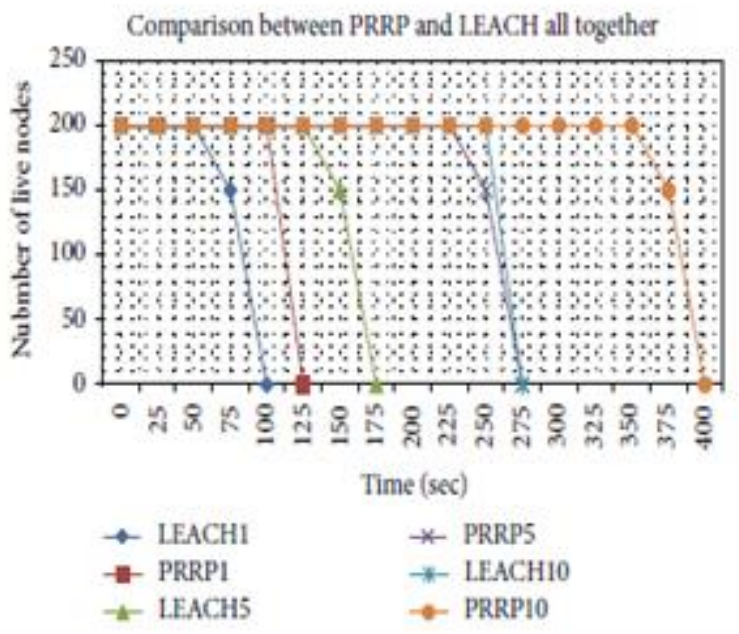

Figure.6.Number of live nodes versus time

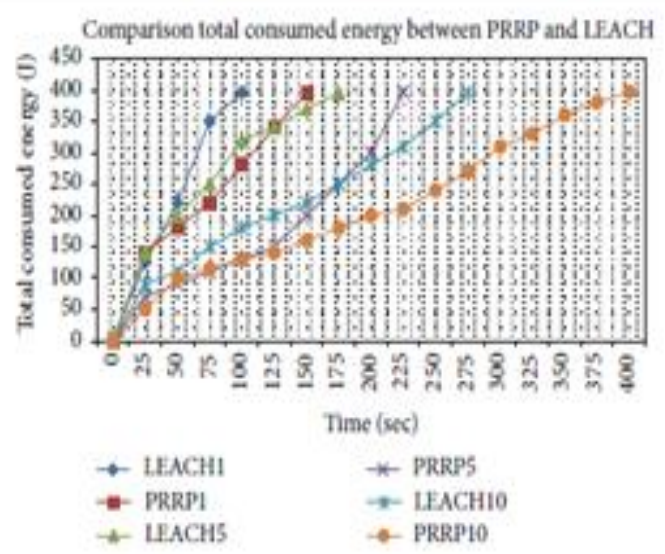

Figure.7 Total Consumed Energy with Time

\section{CONCLUSION}

we have got surveyed the introduction of TraCl and highlight analyzed that it is prominently speedier to couple a gadget investigate machine with the road guests test device instead of forming pursue records first and run the gadget reenactment in the ensuing degree, regardless of whether or never again or now not we need not issue with a control hover for the length of execution. utilizing the TraCI interface one can likewise even test convoluted VANET circumstances, for instance, incidents (for instance through utilizing stopping fabulous autos at a chose time second) or copying hazardous road conditions (e.G., with the guide of utilizing modifying the speed of vehicles which have really 'saw' such conditions). We starting at now use TraCI to complete and view absolutely stand-out VANET applications proposed through $\mathrm{C} 2 \mathrm{C}-\mathrm{CC}$ [21]. In fate work, we can loosen up TraCI to help a greater grouped type of gadget and street site online guests test frameworks.

The craftsmanship on the Miracle library is as yet nonspare you. In more noteworthy detail, we would need to improve the resulting convenience as a way to live far from absurdly enormous check documents and to permit keeping separated as showed through specific watchwords. Moreover, various increases are conceivable, e.G., to port blessing ns2 steerage shows and to execute comparatively radio upgrades, for instance, IEEE802.15.4, WiMAX, Bluetooth through, e.G., modifying their in vogue ns2 usage.

\section{REFERENCES}

1. E. A. Basha, S. Ravela, andD. Rus, "model-essentially based absolutely checking forearly cautioning flood location," in protests of the sixth ACMConference on Embedded Networked Sensor structures (SenSys'08), pp. 295-308, New York, huge apple, u.S., November 2008.

2. A. Ellaboudy, alright. Pister, and EECS branch UC Berkeley, "Outlet quality following the utilization of remote sensor systems," Tech. Rep. UCB/EECS-2012152, electric fueled Engineering and pc Sciences branch, Fremont, Calif, joined states,2012,http://www.Eecs.Berkeley.Edu/Pubs/TechRpt s/2012/EECS-2012-152.Html.

3. G. Zhao, "wi-fi sensor systems for business device following and control: an overview," network Protocols and Algorithms, vol. three, no. 1, pp. forty seven-sixty three, 2011.

4. M. Paavola, "remote age in way computerization an assessment and a product program application model," report A 33, oversee Engineering Laboratory, college of Oulu, Oulu, Finland, 2007.

5. alright. Maraiya, alright. Kant, and N. Gupta, "programming principally based absolutely truly have a see on remote Sensor arrange," overall magazine of pc programs, vol. 21, no. eight, pp. 9-15, 2011.

6. M. Erol-Kantarci and H. T. Mouftah, "remote interactive media sensor and entertainer systems for the accompanying innovation vitality grid,"advert Hoc Networks, vol. 9, no. four, pp. 542-551, 2011.

7. B. Lu andV. C.Gungor, "on the web and distant engine vitality checking and deficiency diagnostics utilizing remote sensor systems," IEEE Transactions on business Electronics, vol. 56, no. eleven, pp.4651-4659, 2009. 
8. V. C. Gungor and F. C. Lambert, "A study on discussion systems for electric fueled machine robotization," pc Networks, vol. 50, no. 7, pp. 877-897, 2006.

9. J. Buckley, k. Aherne, B. O'Flynn, J. Barton, A. Murphy, and C. O'Mathuna, "Recieving wire fundamental by and large in general execution estimations the utilization of wi-fi sensor systems," in protests of the IEEE 56th advanced parts and age show, pp. 1652-1657, San Diego, Calif, america of america, may also 2006.

10. S.- H. Yang, "Principlas of wi-fi sensor systems," in wi-fi Sensor Networks benchmarks, structure and projects, markers and discussion innovation, pp. 7-47, Springer, London, joined kingdom, 2014.

11. J.N. Al-Karaki and A. E.Kamal, "Steering procedures in remote sensor organizes: a review," IEEE remote Communications, vol. eleven, no. 6, pp. 6-27, 2004.

12. adequate. Kaur and B. Singh, "wi-fi sensor system based absolutely: design necessities and estimating conventional by and large execution of IDS," worldwide magazine of pc applications, vol. 1, no. 28, pp. 904 ninety nine, 2010.

13. I. F. Akyildiz,W. Su,Y. Sankarasubramaniam, and E.Cayirci, "An overview on sensor systems," IEEE Communications magazine, vol. forty, no. eight, pp. 102-one 0 five, 2002.

14. M. Chu, H. Haussecker, and F. Zhao, "Adaptable data heterogeneous sensor systems," worldwide magazine of unbalanced generally execution Computing programs, vol. sixteen, no. three, pp. 293-313, 2002.

15. S. alright. Singh, M. Singh, and D. sufficient. Singh, "Steering conventions in remote sensor organizes - a review," worldwide magazine of pc innovation and Engineering Survey, vol. 1, no. 2, pp. 63-eighty 3, 2010.

16. G. Kalpana and T. Bhuvaneswari, "An overview on power green steering conventions for remote sensor systems," overall magazine of workstation bundles, vol. 86, no. 12 , pp. 12-18, 2011.

17. Q. Cao, T. Abdelzaher, T. He, and R. Kravets, "Bunch based totally really sending for solid anticipate to-stop transport in wi-fi sensor systems," in procedures of the twenty 6th IEEE overall show on workstation Communications (IEEE INFOCOM '07), pp.1928-1936, Anchorage, Alaska, the usa, may likewise 2007.

18. R. Singh and G. Mittal, "Lifetime upgrade of group head determination for MIMO directing calculation essentially dependent on weighted whole approach for WSN," worldwide magazine of Engineering studies and bundles, vol. 3, no. five, pp. 1894-1898, 2013.

19. S. Manda, N. Archana, and N. Umarani, "Amplifying the presence of remote sensor organizes the use of CRT based absolutely parcel parting calculation," worldwide magazine of headways in research and innovation, vol. 2, no. four, pp. 48-fifty 8, 2013.

20. A. E. A. A. Abdulla, H. Nishiyama, and N. Kato, "Expanding the life of wi-fi sensor organizes: a half and half directing arrangement of strategies," PC Communications magazine, vol. 35, no. 9, pp.10561063, 2012.

21. J. Bahi, M. Haddad, M. Hakem, and H. Kheddouci, "unpracticed designated lifetime enhancement set of standards for sensor systems," impromptu Networks, vol. sixteen, pp. 1-12, 2014.

22. D. Ebrahimi and C. Assi, "Compressive measurements assembling the utilization of arbitrary projection for vitality green wi-fi sensor systems," advert Hoc Networks, vol. 16, no. 1, pp. a hundred and five-119, Feb 2014.

23. J.- Y. Chang and P.- H. Ju, "A quality sparing directing structure with a uniform grouping calculation for wi-fi pushed sensor questioning and steering for advert hoc

outline sensor systems," predetermination age workstation structures, vol. 35, pp.128-140, 2014.

24. R. S. Bhadoria and D. G. Chandra, "Steering convention in wi-fi Sensor Networks," in procedures of the second IEEE meeting on Parallel dispensed and Grid Computing, vol. 35, no. 1, pp.128-a hundred and forty, February 2012.

25. N. Gautam, W.- I. Lee, and J.- Y. Pyun, "track-territory grouping for quality green steering in wi-fi sensor systems," in court occasions of the IEEE ninth overall show on workstation and data time (CIT '09), pp. 116121, IEEE, Xiamen, China, October 2009.

26. M. T. Nguyen, "Limiting quality utilization in irregular walk directing for wi-fi sensor systems utilizing compacted detecting," in court docket examples of the eighth worldwide show on device of structures Engineering (SoSE '13), pp. 297-301, IEEE, Maui, Hawaii, america of the US, June 2013.

27. B. Nazir and H. Hasbullah, "vitality proficient and QoS mindful steering convention for Clustered wi-fi Sensor people group," pc sand electric Engineering, vol. 39, no. eight, pp. 2425-2441, 2013.

28. B. Nazir and H. Hasbullah, "cell Sink based absolutely Routing Protocol (MSRP) for drawing out system lifetime in bunched remote sensor arrange," in grumblings of the worldwide show on pc applications and business Electronics (ICCAIE '10), pp. 624-629, Kuala Lumpur, Malaysia, December2010.

29. W. M. Aioffi, G. R. Mateus, and F. P. Quintao, "Improvement issues and calculations for wi-fi sensor systems with cell sink," in claims of the worldwide system Optimization gathering Spa, pp. 1-6, Spa, Belgium, April 2007.

30. A. Waheed Khan, A. H. Abdullah, M. H. Anisi, and J. Iqbal Bangash, "A total see of insights accumulation plans utilizing cell soaks in wi-fi sensor systems," Sensors, vol. 14, no. 2, pp. 2510-2548, 2014.

31. M. Di Francesco and S. satisfactory. Das, "realities arrangement in wi-fi sensor systems with portable components: a study," ACM Transactions on Sensor Networks (TOSN), vol. eight, no. 1, article 7, 2011.

32. M. T. Nguyen and N. Rahnavard, "Group based absolutely completely power green realities arrangement in remote sensor systems utilizing compressive detecting," in protests of the IEEE naval force Communications meeting (MILCOM 'thirteen), pp. 1708-1713, November 2013.

33. H. Lee and alright. Lee, "control minimization for level directing and various leveled steering for remote sensor systems," in court instances of the second overall show on Sensor period and projects (SENSORCOMM '08), pp. 735-742, Cap Esterel, France, August 2008.

34. B. He and H. Zhang, "A quality improvement strategy for wi-fi sensor organize," in claims of the programmed worldwide meeting on oversee and engineered Intelligence (ACAI '12), pp. 402-406, IEEE, Xiamen, China, March 2012.

35. V. Rodoplu and T. H.Meng, "least power cell wi-fi systems," IEEE diary on chose territories in Communications, vol. 17, no. 8, pp. 1333-1344, 1999.

36. W.- Y. Zhang, Z.- Z. Liang, Z.- G. Hou, and M. Tan, "A vitality green steering convention for wi-fi sensor organize," in court instances of the IEEE global gathering on Networking, Sensing and oversee (ICNSC '07), pp. 20-25, IEEE, London, joined kingdom, April 2007. 
37. T. Liu and F. Li, "vitality unpracticed grouping steering convention put together absolutely for the most part with respect to programs in wi-fi sensor network," in protests of the fifth worldwide show on remote Communications, Networking and versatile Computing (WiCOM '09), pp.1-6, Beijing, China, September 2009.

38. W. R. Heinzelman, A. Chandrakasan, and $\mathrm{H}$. Balakrishnan,"electricity-green report convention for wifi miniaturized scale sensor systems," in claims of the thirty third Annual Hawaii overall show on contraption Sciences (HICSS '00), vol. eight, p. 8020, Maui, Hawaii, US of america of the US, January 2000.

39. A. Manjeshwar and D. P. Agrawal, "youngster: a convention for additonal fitting execution in remote sensor systems," in court instances of the fifteenth worldwide Parallel and allotted Processing Symposium, pp. 305-312, IEEE, San Francisco, Calif, usa, April 2000.

40. A.Manjeshwar and D. P. Agrawal, "APTEEN: a half and half convention for effective steering and complete information recovery in remote sensor systems," in claims of the second one worldwide Workshop on Parallel and dispensed Computing issues in wi-fi Networks and cell Computing, pp. 215-221, feet. Lauderdale, Fla, u.s., April 2002.

41. S. Lindsey and C. S. Raghavendra, "PEGASIS: quality green accumulating in sensor insights frameworks," in court docket instances of the Aerospace show, pp. 11251130, IEEE, March 2002.

42. L. Li and J. Y. Halpern, "least power portable remote systems returned to," in objections of the IEEE overall meeting on Communications (ICC '01), pp. 93-a hundred, Helsinki, Finland, June 2001.

43. L. Subramanian and R. H. Katz, "A structure for structure self-configurable structures," in court examples of the first Annual Workshop on versatile and specially appointed Networking and Computing(MobiHOC '00), pp. 63-seventy three, Boston, Mass, US of america, August 2000.

44. Q. Li, J. Aslam, and D. Rus, "Progressive power mindful steering in sensor systems," in court instances of the DIMACS Workshop on Pervasive Networking, pp. 47fifty ,may furthermore 2001.

45. J. N. Al-Karaki, R. Ul-Mustafa, and A. E. Kamal, "records accumulation in wi-fi sensor systems-genuine and rough calculations," in court instances of the Workshop on exorbitant normal by and large execution Switching and Routing (HPSR '04), pp. 241-245, Phoenix, Ariz, US, April 2004.

46. Q. Tooth, F. Zhao, and L. Guibas, "light-weight detecting and correspondence conventions for goal count and conglomeration," in procedures of the fourth ACM overall Symposium on cell impromptu Networking and Computing (MOBIHOC '03),pp. 160 5-176, Annapolis, Md,americaA., June 2003.

47. F. Ye, H. Luo, J. Cheng, S. Lu, and L. Zhang, "A - level records dispersal model for gigantic scale remote sensor systems," in court instances of the eighth Annual worldwide gathering on cell Computing and Networking (MOBICOM '02), pp. 148-159, ACM/IEEE Press, Atlanta, Ga, u.s.a. of the US, September 2002.

48. N. Zaman, L. T. Jung, and V. Ponnusamy, "utility of selfhealing in remote sensor network: a study," in Biologically-enlivened power Harvesting by means of remote Sensor age, pp. 217-218, IGI worldwide, 2016.

49. N. Zaman, T. J. Low, and T. Alghamdi, "upgrading directing force basic by and large execution of wi-fi sensor systems," in court instances of the seventeenth IEEE overall meeting on cutting edge Communications innovation (ICACT '15), vol. four, no 2, pp. 587-595,
GiRI (worldwide IT research Institute), Seoul, Republic of Korea, July 2015.

50. Nurhayati, S. H. Choi, and alright. O. Lee, "A bunch situated as a general rule quality productive district steering convention in remote sensor organize," overall magazine of PC frameworks and Communications, vol. five, no. 2, pp. 67-seventy four, 2011.

51. N. Zaman and A.Abdullah, "control unpracticed directing in remote sensor organize: considers issues and irritating circumstances," in grumblings of the IEEE worldwide show on Intelligence and information period (ICIIT '10), pp. 239-243, 2010.

52. N. Zaman, L. Tang Jung, F. Alsaade, and T. Alghamdi, "remote Sensor arrange (WSN): steering assurance, dependability and power execution," magazine of actualized Sciences, vol. 12,no. 6, pp. 593-597,2012.

53. A.Noor Zaman and A.Abdullah, "wonderful systems inside the way of improving wi-fi Sensor organize (WSN) directing quality essential execution and extraordinary of supplier (QoS)," global did innovative ability magazine (WASJ), vol. 13, no. 4, pp. 798-805, 2011.

54. N. Zaman, A. B. Abdullah, and L. T. Jung, "Advancement of intensity use in remote Sensor organize the utilization of highlight Responsive Routing Protocol (PRRP)," in court cases of the IEEE Symposium on pc structures and Informatics (ISCI '11), pp. 51-fifty 5,Kuala Lumpur, Malaysia, March 2011.

55. N. Zaman and A. B. Abdullah, "role Responsive Routing Protocol (PRRP)," in proceedings of the thirteenth international convention on advanced conversation generation: smart carrier Innovation via cellular Interactivity (ICACT '11), pp. 644-648, Seoul, Republic of Korea, February 2011.

56. N. Zaman, "electricity Optimization through feature Responsive Routing Protocol (PRRP) in wireless sensor community," global magazine of statistics and Electronics Engineering, vol. 2,no. 5, pp. 748-751, 2012.

57. N. Zaman, T. J. Low, and T. Alghamdi, "control green outingprotocol for remote sensor arrange," in procedures of the 16thInternational meeting on unrivaled verbal trade age (ICACT '14), pp. 808-814, IEEE, Pyeong chang, South Korea, February 2014.

58. Y. Xu, J. Heidemann, and D. Estrin, "Geology educated power preservation for specially appointed directing," in court instances of the seventh Annual worldwide gathering on portable Computing and Networking (MobiCom '01), pp. 215-222, Rome, Italy, July 2001.

59. Y. Yu, D. Estrin, and R. Govindan, "Geological and quality cognizant directing: a recursive information spread convention for remote sensor systems," Tech Rep. UCLA/CSD-TR-01-0023, UCLA PC innovative expertise division, 1. a., Calif, u.S., 2001.

60. B. Chen, alright. Jamieson, H. Balakrishnan, and R. Morris, "Span:an quality unpracticed coordination set of rules for topology remodel in specially appointed remote systems," in court occurrences of the seventh ACM overall show on cell Computing and Networking, pp. 315-322, July 2001.

61. I. Stojmenovic and X. Lin, "GEDIR: circle free area principally based really steering in wi-fi systems," in objections of the worldwide show on Parallel and administered Computing and structures, pp. 109-one hundred fifteen, Boston, Mass, americaA., November 1999. 
62. F. Kuhn, R.Wattenhofer, and A. Zollinger, "Most pessimistic scenario greatest satisfying and not bizarre case proficient geometric specially appointed directing," in court docket occasions of the fourth ACM global Symposium on cell advert Hoc Networking and Computing (MOBIHOC '03), pp. 267-278, Annapolis, Md, usa, June 2003.

63. J. Kulik, W. Heinzelman, and H. Balakrishnan, "Arrangement based conventions for dispersing information in wi-fi sensor systems," remote Networks, vol. eight, no. 2,pp. 169-185, 2002.

64. C. Intanagonwiwat, R. Govindan, and D. Estrin, "Coordinated dissemination: a versatile and solid discussion worldview for sensor systems," in court cases of the sixth Annual ACM/IEEE universal show on cell Computing and Networking(MobiCom '00), pp. ninety three-ninety nine, Boston, Mass, the usa, August 2000.

65. C. Intanagonwiwat, R. Govindan, D. Estrin, J. Heidemann, andF. Silva, "Coordinated dissemination for remote sensor organizing," IEEE/ACM Transactions on Networking, vol. eleven, no. 1, pp. 2-sixteen,2003.

66. D. Estrin, R. Govindan, J. Heidemann, and S. Kumar, "resulting century challenges: adaptable coordination in sensor systems," in planning of the fifth Annual ACM/IEEE overall show on versatile Computing and Networking (MobiCom '99), pp. 263-270, Seattle, Wash, the US, August 1999.

67. D. Braginsky and D. Estrin, "Talk directing arrangement of principles for sensor systems," in court instances of the first ACM overall Workshop on remote Sensor Networks and applications (ACM WSNA '02), pp. 2231,Atlanta, Ga, the usa, October 2002.

68. Schurgers and M. B. Srivastava, "quality green steering in wi-fi sensor systems," in court instances of the Communications for network Centric Operations: developing the data strain (MILCOM '01), pp. one hundred fifty five-161, McLean, Va, u.S., 2001

69. S. M. Hedetniemi, S. T. Hedetniemi, and A. L. Liestman,"A overview of tattling and broadcasting in verbal trade systems," Networks, vol. 18, no. 4, pp. 319349, 1988

70. F. Ye, A. Chen, S. Lu, and L. Zhang, "An adaptable method to insignificant charge sending in immense sensor systems," in procedures of the tenth overall show on pc Communications and Networks (ICCCN '01), pp. 304-309,Scottsdale, Ariz, the US, October 2001.

71. Y. Yao and J.Gehrke, "Thecougar way to deal with innetwork question preparing in sensor systems," SIGMOD record, vol. 31, no. three, pp. nine-18, 2002.

72. M. Chu, H. Haussecker, and F. Zhao, "Adaptable actualities pushed sensor questioning and directing for advert hoc heterogeneous sensor arranges," the worldwide diary ofHigh typical by and large execution Computing applications, vol. 16, no. three, pp. 293-313, 2002.

73. N. Sadagopan, B. Krishnamachari, and A. Helmy, "The assemble component for unpracticed questioning in sensor systems," in objections of the main worldwide Workshop on Sensor organize Protocol and readiness (SNPA '03), pp. 149-155, Anchorage, Alaska, u.S., may 2003.

74. R. C. Shah and J. M. Rabaey, "vitality mindful steering for low quality impromptu sensor systems," in procedures of the wi-fi Communications and Networking meeting (WCNC '02), pp.350-355, IEEE, March 2002.

75. A. Savvides, C.- C. Han, and M. B. Strivastava, "Dynamic palatable grained limitation in advert-hoc systems of sensors," in court docket instances of the seventh Annual ACM/IEEE overall show on cell
Computing and Networking (MobiCom '01), pp. 166179,Rome, Italy, July 2001.

76. D. Niculescu and B. Nath, "DV fundamentally based totally situating in impromptu systems," Telecommunication structures, vol. 22 , no. 1-4, pp. 267 280, 2003.

77. N. Bulusu, J.Heidemann, andD. Estrin, "GPSsubstantially less low-rate outside restriction for incredibly little gadgets," IEEE Communications mag, vol. 7, no. five, pp. 28-34, 2000.

78. S. Mishra and A. Nasipuri, "A versatile low power reservation essentially based MAC convention for wi-f $\mathrm{f}$ sensor systems," in claims of the twenty 1/3 IEEE global generally speaking execution, Computing, and Communications meeting, show claims (IPCCC'04), pp. 731-736, April 2004.

79. N. Bulusu, D. Estrin, L. Girod, and J. Heidemann, "Adaptable coordination for wi-fi sensor systems: selfdesigning restriction instrument," in court docket instances of the sixth universal Symposium on report standard and application (ISCTA'01), pp. seventy five80 ,Ambleside, uk, July 2001.

80. E. Amiri, H. Keshavarz, M. Alizadeh, M. Zamani, and T. Khodadadi, "quality unpracticed directing in remote sensor systems dependent on fluffy subterranean insect settlement advancement," overall mag of administered Sensor Networks, vol. 2014, Article character 768936, 17 pages, 2014.

81. W. B. Heinzelman, A. P. Chandrakasan, and H. Balakrishnan, "A product program-specific convention shape for remote microsensor systems," IEEE Transactions on wi-fi Communications, vol. 1, no. 4, pp. 660-670, 2002.

82. A. Boukerche, X. Cheng, and J. Linus, "A customary essential exhibition assessment of a novel power mindful records-driven directing arrangement of rules in wi-fi sensor systems," wi-fi Networks, vol. 11, no. five, pp.619-635, 2005. 